PHYSICAL REVIEW D 95, 049903(E) (2017)

\title{
Erratum: Energy-momentum correlations for Abelian Higgs cosmic strings [Phys. Rev. D 93, 085014 (2016)]
}

David Daverio, Mark Hindmarsh, Martin Kunz, Joanes Lizarraga, and Jon Urrestilla

(Received 3 February 2017; published 22 February 2017)

DOI: $10.1103 /$ PhysRevD.95.049903

In our paper we presented new results for the energy-momentum unequal time correlators (UETCs) of Abelian Higgs cosmic strings. The new modeling includes contributions from simulations of strings with constant physical width $(s=1)$, improving on previous simulations which used constant comoving width $(s=0)$. The dramatically increased volume also allowed the UETCs to be measured over greater conformal time ratios and a wider range of wave number.

In the paper, we neglected to take into account the fact that covariant conservation of the energy-momentum (EM) tensor constrains the superhorizon behavior of the vector correlators. The constraints come from the fact that EM conservation links the two vector parts of the strings' EM tensor through:

$$
\dot{T}_{0 i}+H T_{0 i}+i k_{j} T_{i j}=0
$$

There is no constraint on the spatial parts $T_{i j}$, so as the strings are created by a random causal process (the phase transition), the power spectral density is just white noise, or $\left\langle\left|T_{i j}\right|^{2}\right\rangle \propto k^{0}$. Energy momentum conservation means that the spectral density of $T_{0 i}$ must be consistent with the white noise behavior of $T_{i j}$ : hence $\left\langle\left|T_{0 i}\right|^{2}\right\rangle \propto k^{2}$.

Our fitting in Appendix B of the paper did not consider this constraint and thus the vector equal time correlator (ETC) does not follow the behavior dictated by it at low- $k \tau$. This erratum aims to highlight that the decay of the vector UETCs at superhorizon scales must be corrected to be $\sim z^{2}$ where $z=k \sqrt{\tau \tau^{\prime}}$.

We apply this correction to the vector correlator obtaining the values corresponding to the $\mathbf{k}$ bins in the range $0.17<z_{\xi} \exp \left(-\left|\ln \left(r_{\xi}\right)\right|\right)<1.29$ by extrapolating the value of the correlator at the upper end of the range. Using the notation of the published paper,

$$
C_{\mathrm{vv}}^{(\mathrm{tot}, \xi)}\left(z_{\xi}, r_{\xi}\right)=C_{\mathrm{vv}}^{(\mathrm{tot}, \xi)}\left(z_{\xi}^{*}, r_{\xi}^{*}\right)\left(\frac{z_{\xi}^{2}}{z_{\xi}^{* 2}}\right),
$$

where $z_{\xi}^{*} \exp \left(-\left|\ln \left(r_{\xi}^{*}\right)\right|\right)=1.29$.

Only vector perturbations are affected by this constraint, hence the assumptions and extrapolations applied to other perturbation correlators are not affected.

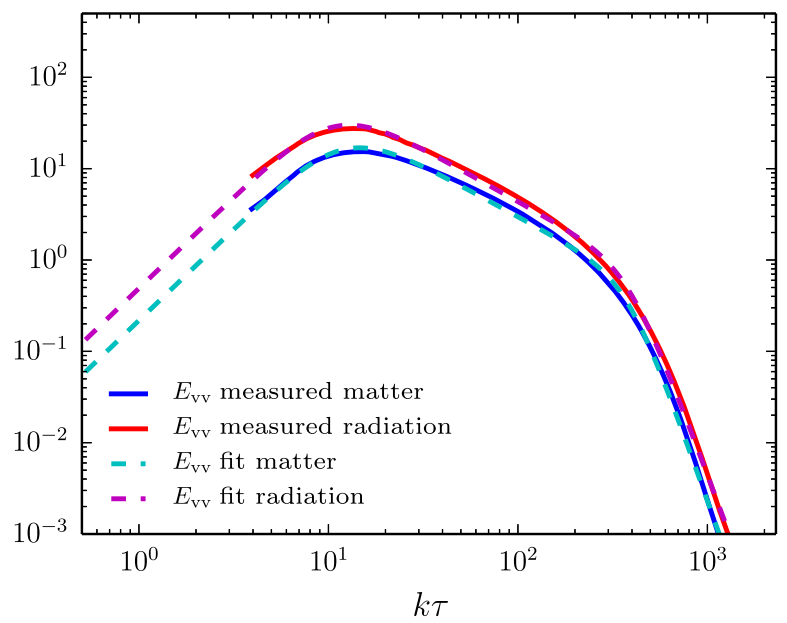

FIG. 1. Fits of the ETC $E_{\mathrm{vv}}(k \tau)$ of the vector source function at the reference time to the function given in Eq. (3). The solid curves show the measured ETCs and the dashed curves the fits, for the numerical values given in Table I. 
TABLE I. The numerical values of the fits of the vector ETC shown in Fig. 1 to Eq. (3).

\begin{tabular}{lcc}
\hline \hline Parameters & $E_{\mathrm{vv}}$ mat & $E_{\mathrm{vv}}$ rad \\
\hline$a_{2}$ & 0.22 & 0.49 \\
$z_{p}$ & 12.2 & 10.8 \\
$d$ & 1.14 & 1.16 \\
$w$ & 384 & 387 \\
$\delta$ & 4.7 & 4.4 \\
\hline \hline
\end{tabular}

The correction only affects the $\mathrm{CMB}$ vector power spectrum at $\ell \sim 30$, thus Planck constraints will not be affected. In order to support this statement we have tested some cases, and checked that there is no difference to the constraints on the string tension $G \mu$ presented in [1].

Here we give the fit results presented in Appendix B taking into account the constraint discussed above, i.e., with low $x$ behavior of the ETC $E(x)$ going as $x^{2}$. The fitting formula (B1) now becomes

$$
E(x)=\frac{a_{2} x^{2}}{\left(1+\left(x / z_{p}\right)^{2+d}\right)\left(1+(x / w)^{\delta}\right)} .
$$

The numerical values reported in Table $\mathrm{X}$ change slightly, but overall remain consistent with the previous fit. We give them in Table I. We also show in Fig. 1 the (updated) relevant panel of Fig. 17 of the published paper.

[1] J. Lizarraga, J. Urrestilla, D. Daverio, M. Hindmarsh, and M. Kunz, J. Cosmol. Astropart. Phys. 10 (2016) 042. 